the presence of these specific DHSs correlated with the upregulation of their associated genes.

The authors next determined, by digital footprinting, whether transcription factor binding motifs within the 20 AML-specific DHS clusters were occupied, noting that motif occupancy within these samples was also AML-subtype specific. AML subtypes showed mixed occupancy patterns when compared with normal myeloid progenitor cells, suggesting that their myeloid differentiation trajectories went 'sideways' instead of following the normal hierarchy.

Next, CREs were linked to their respective promoters using promoter-capture chromosomal structure analysis (CHi-C). AML-subtype-specific CREs exhibited differences in locus-specific CRE promoter interactions between AML subtypes and control cells. These differences correlated with the differential expression of gene groups with a broad range of predicted functions.

Integrating DNase I footprinting, gene expression and $\mathrm{CHi}-\mathrm{C}$ data to link CREs to their promoters

could contribute to PDL1-dependent control of $\mathrm{CD} 8^{+} \mathrm{T}$ cells, the authors identified increased expression of PDL1 in lymphatic endothelial cells (LECs) and blood endothelial cells from B16F10 and YUMMER1.7 tumours relative to naive skin. Furthermore, PDL1 expression exclusively in tumour-associated LECs could be upregulated by inducing a tumour antigen-specific $\mathrm{CD} 8^{+} \mathrm{T}$ cell response in mice. In agreement with this observation, in vitro-activated but not naive $\mathrm{CD}^{+} \mathrm{T}$ cells could increase PDL1 expression on mouse immortalized LECs, an effect that could be inhibited by the presence of an IFN $\gamma$-blocking antibody and suggestive of indirect activation of LECs in response to IFN $\gamma$ secreted by antigen-specific T cells. Importantly, in human primary melanoma samples, lymphatic vessels and CD8 ${ }^{+}$ T cells were both localized to the peritumoural stroma and in close proximity to one another, consistent with the IFN $\gamma$ - and PDL1-mediated crosstalk between tumour-associated lymphatic vessels and infiltrating CTLs being functionally important. revealed that all of the AML subtypes studied had specific gene regulatory network (GRN) signatures and differed in the signalling pathways that were predicted to be activated. In the AML subtypes harbouring $t(8 ; 21)$ or a FLT3-ITD and NPM1 alteration, the importance of several key GRN-specific transcription factors was validated in cell culture, where their short hairpin RNA-mediated knockdown blocked cell proliferation. Furthermore, the GRN for both of these subtypes included multiple genes bound by the AP-1 family of transcription factors, suggesting that these transcription factors have an important role within the GRN. This idea was validated in mice, in which the inhibition of AP-1 activity blocked leukaemia development.

Overall, this study presents a wealth of data on AML-subtypespecific transcriptional and signalling networks, which may include novel therapeutic targets.

Katharine H. Wrighton

ORIGINAL ARTICLE Assi, S. A. et al. Subtypespecific regulatory network rewiring in acute myeloid leukemia. Nat. Genet. https://doi.org/ 10.1038/s41588-018-0270-1 (2018)

To demonstrate that IFN $\gamma$ signalling through lymphatic vessels was suppressing intratumoural T cell activity in melanoma, the authors implanted YUMMER1.7 cells into mice whose LECs were unresponsive to IFN $\gamma$ due to a lymphatic-specific deletion of the IFN $\gamma$ receptor (IFN $\gamma$ R). When compared with WT mice, mice with a genetic deficiency of the IFN $\gamma \mathrm{R}$ showed inhibition of tumour growth and extended survival. In addition, these in vivo effects were dependent on $\mathrm{CD} 8^{+}$ $T$ cells and associated with a lack of PDL1 upregulation in tumour-resident LECs.

This study proposes that lymphatic vessels, through PDL1 and IFN $\gamma$, undergo adaptive immune resistance during tumour progression and could likely contribute to patient response to PDL1-mediated immune checkpoint blockade.

Anna Dart

ORIGINAL ARTICLE Lane, R. S. et al. IFN $\gamma$ activated dermal lymphatic vessels inhibit cytotoxic T cells in melanoma and inflamed skin. J. Exp.Med.https://doi.org/10.1084/jem.20180654 (2018)

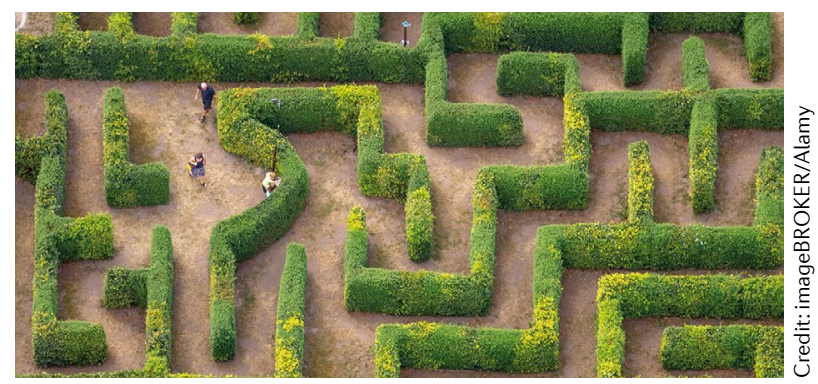

GENOMICS

\section{Cancer chromatin accessed}

The Cancer Genome Atlas (TCGA) has been instrumental in studying genomic and epigenomic aberrations in a multitude of different human cancers. Corces, Granja et al. now report a rich data resource of accessible chromatin regions for 23 cancer types that provides insight into the landscape of active regulatory DNA elements and transcriptional regulation in human tumours.

A total of 410 tumour samples were used as input for assay for transposase-accessible chromatin using sequencing (ATAC-seq), which identified more than 500,000 pan-cancer peaks of chromatin accessibility. These peaks overlapped with promoter and enhancer regions defined by chromatin immunoprecipitation followed by sequencing (ChIP-seq) in the ChromHHM database, as expected. Distal elements showed stronger cancer type specificity, whereas proximal, promoter regulatory elements showed similar patterns across cancers.

Based on the genome-wide ATAC-seq patterns, cancer types were clustered into different groups, and cluster-specific peak sets were observed to be enriched for relevant transcription factor (TF) motifs as well as genetic variants identified in genome-wide association studies for these cancers. New cancer subtypes could be defined on the basis of these chromatin accessibility patterns.

Correlating the ATAC-seq peaks with matched RNA sequencing data, the authors were able to predict peak-to-gene links, both across all cancers and specifically for breast cancer. To validate a subset of these links, a CRISPR interference (CRISPRi) approach was used to introduce heterochromatin signatures at select distal peaks. Peaks targeted in this way were associated with a decrease in expression of the linked gene, located kilobases or megabases away. These assays also emphasized the cell type and cancer type specificity of the peak-gene relationships, for example for BCL2 in luminal-like but not basal-like breast cancer.

Complementary to this unique resource, future studies will be crucial to delineate the contribution of cellular composition of the ATAC-seq signal, to include (matched) healthy tissues and to add additional genomic data such as 3D chromosome conformation to enable researchers to home in on the causative genomic regions in a wide range of human cancers at the full level of complexity of the tumour tissue.

Michelle Trenkmann, Associate Editor, Nature Communications This article is modified from the original in Nat. Rev. Genet. (https://doi.org/10.1038/s41576-018-0075-1).

ORIGINAL ARTICLE Corces, M. R. et al. The chromatin accessibility landscape of primary human cancers. Science 362, eaav1898 (2018) 